sonic Unsteady Lifting Surfaces," AIAA Journal, Vol. 22, No. 2, 1984 , pp. $179-186$

${ }^{8}$ Tewari, A., "Nonplanar Doublet-Point Method in Supersonic Three-Dimensional, Unsteady Aerodynamics," M.S. Thesis, Univ. of Missouri-Rolla, Rolla, MO, 1988.

'Tewari, A., "New Acceleration Potential Method for Supersonic Unsteady Aerodynamics of Lifting Surfaces, Further Extension of the Nonplanar Doublet Point Method, and Nonlinear, Nongradient Optimized Rational Function Approximations for Supersonic, Transient Response Unsteady Aerodynamics," Ph.D. Dissertation, Univ. of Missouri-Rolla, Rolla, MO, 1992.

${ }^{10}$ Luenberger, D. G., Introduction to Linear and Nonlinear Programming, Addison-Wesley, Reading, MA, 1973.

\section{Neutrally Reinforced Holes in Symmetrically Laminated Plates}

\author{
E. Senocak* and A. M. Waas $\dagger$ \\ University of Michigan, Ann Arbor, Michigan 48109
}

\section{Introduction}

$\mathbf{S}$ INCE laminated composite plates with openings are widely used structural elements in many engineering applications, the analysis of such structures has been done by numerous researchers. The stress and strain state around the openings have been presented for a variety of problems. A large number of problem solutions are presented in the monographs by Lekhnitskii ${ }^{1,2}$ and Savin. ${ }^{3}$ In the formulation of these problems, the material constitutive law, the geometry, loading, and boundary conditions are assumed to be given and the stress and strain state are computed.

In a particular class of structural optimization, the geometry of the body is obtained as a solution to a particular problem, for known states of stress, strain, boundary condition and appropriate constraints (sometimes called inverse problems for some specific cases). In the latter category, either stress concentrations are reduced (optimized) by determining the shape of the openings (harmonic holes), or stress states in the cut structures with reinforcement are maintained unchanged to that of the uncut structures (neutral holes).

All previous solutions, ${ }^{3-5}$ for neutral holes have been obtained for elastic, isotropic sheets under planar states of stress. In a previous paper, ${ }^{6}$ the methodology outlined in Ref. 1 was extended to symmetrically laminated composite plates which are under planar loading. In the present work, the case of a symmetrically laminated plate subjected to pure bending moments is considered. It must be noted that the notion of a neutral hole in the context of plate flexure assumes a new meaning. Here, the purpose is to introduce a reinforced cutout into a plate that will maintain the same moment and curvature distributions as of the uncut plate throughout.

Received Feb. 19, 1992; presented as Paper 92-2486 at the AIAA/ ASME/ASCE/AHS/ASC 33rd Structures, Structural Dynamics and Materials Conference, Dallas, TX, April 13-15, 1992; revision received Sept. 1, 1992; accepted for publication Sept. 10, 1992. Copyright (C) 1992 by E. Senocak and A. M. Waas. Published by the American Institute of Aeronautics and Astronautics, Inc., with permission.

${ }^{*}$ Graduate Research Assistant, Department of Aerospace Engineering. Student Member AIAA.

†Assistant Professor of Aerospace Engineering, Department of Aerospace Engineering. Senior Member AIAA.

\section{Formulation}

\section{Laminated Plate Under Bending}

Let a Cartesian coordinate system be located in the midplane of the laminate, about which the plate is laminated symmetrically, such that the $x-y$ plane coincides with the lamination plane and the $z$ axis is perpendicular to that plane. Under "classical laminate theory" assumptions, the lateral force and moment equilibrium equations are the following 8 :

$$
\begin{array}{r}
\frac{\partial Q_{x}}{\partial x}+\frac{\partial Q_{y}}{\partial y}+q=0 \\
\frac{\partial M_{x}}{\partial x}+\frac{\partial M_{x y}}{\partial y}=Q_{x} \\
\frac{\partial M_{y}}{\partial y}+\frac{\partial M_{x y}}{\partial x}=Q_{y}
\end{array}
$$

Here, $M_{x}, M_{y}$, and $M_{x y}$ are the moment resultants (moment per unit length), $Q_{x}, Q_{y}$ are the shear forces per unit length, and $q$ is the lateral surface force per unit area acting on the laminate. From Eq. (1) the following can be deduced in the absence of a lateral force $q$. In this way, the equilibrium is represented with one equation:

$$
\frac{\partial^{2} M_{x}}{\partial x^{2}}+\frac{\partial^{2} M_{y}}{\partial y^{2}}+2 \frac{\partial^{2} M_{x y}}{\partial x \partial y}=0
$$

The reinforcing member is assumed as a beam element which has flexural as well as twisting rigidity. With reference to Figs. 1 and 2 the out-of-plane force and moment equilib-

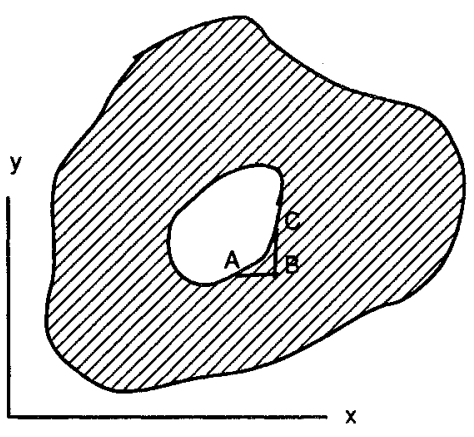

Fig. 1 Plate with a cutout.

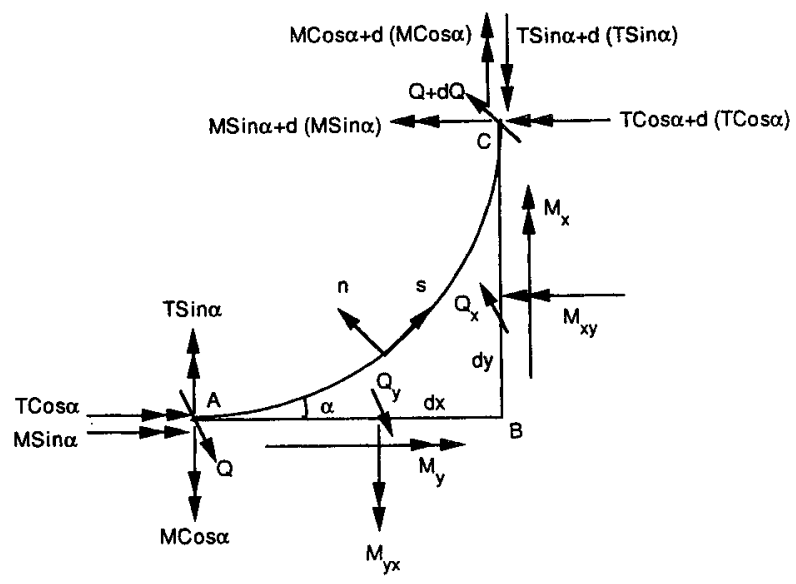

Fig. 2 Moments and out-of-plane forces acting on the element ABC. 
rium equations for a small portion of the laminate adjacent to the cutout and including the reinforcement are

$$
\begin{aligned}
\mathrm{d} Q & =Q_{y} \mathrm{~d} x-Q_{x} \mathrm{~d} y \\
\mathrm{~d}(T \cos \alpha+M \sin \alpha) & =M_{y} \mathrm{~d} x+\left(Q-M_{x y}\right) \mathrm{d} y \\
\mathrm{~d}(T \sin \alpha-M \cos \alpha) & =M_{x} \mathrm{~d} y+\left(-M_{x y}-Q\right) \mathrm{d} x
\end{aligned}
$$

where $M$ and $T$ are the total bending and twisting moments in the reinforcement, respectively, and $Q$ is the total shearing force in the reinforcement.

To proceed with the solution of Eq. (3), first consider Eq. (1) and introduce the functions $\varphi$ and $\psi$ defined as below, which satisfy the equilibrium Eq. (1):

$$
\begin{array}{r}
M_{y}=\frac{\partial \varphi}{\partial x} \\
Q-M_{x y}=\frac{\partial \varphi}{\partial y} \\
M_{x}=\frac{\partial \psi}{\partial y} \\
-M_{x y}-Q=\frac{\partial \psi}{\partial x}
\end{array}
$$

From Eqs. (4) and (5) $M_{x y}$ and $Q$ are to be found as

$$
\begin{gathered}
M_{x y}=-\frac{1}{2}\left(\frac{\partial \varphi}{\partial y}+\frac{\partial \psi}{\partial x}\right) \\
Q=\frac{1}{2}\left(\frac{\partial \varphi}{\partial y}-\frac{\partial \psi}{\partial x}\right)
\end{gathered}
$$

Upon substituting Eqs. (4) and (5) into Eq. (3), and then integration of the last two moment equations, gives the following:

$$
\begin{aligned}
& T \sin \alpha-M \cos \alpha=\psi+e \\
& T \cos \alpha+M \sin \alpha=\varphi+f
\end{aligned}
$$

where $e$ and $f$ are constants of integration.

\section{Reinforcement Kinematics}

In this section, we present the solution for the shape of the hole and reinforcement distribution incorporating kinematics of the reinforcing member. This is different to that of the planar loading case, ${ }^{4,5}$ in which the determination of the shape and reinforcement distribution are uncoupled. An exact solution is obtained within classical laminate theory assumptions. Consider a small element of laminate including a portion of the reinforcement. Then (see Fig. 2) the equilibrium of this element reduce to Eq. (3), whose solution is

$$
\begin{aligned}
T & =\psi \sin \alpha+\varphi \cos \alpha \\
M & =\varphi \sin \alpha-\psi \cos \alpha
\end{aligned}
$$

The constants of integration $e$ and $f$ are chosen to be zero without loss of generality. The twisting and bending moment in the reinforcement are related to the reinforcement twist

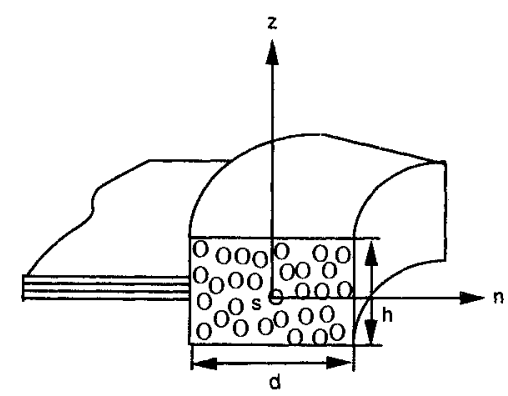

Fig. 3 Coordinates used to define reinforcement properties.

and reinforcement curvature, respectively (the constitutive equations for the reinforcement). These are

$$
\begin{aligned}
T & =G_{n z} Z \bar{\kappa}_{s n} \\
M & =E_{s} I \bar{\kappa}_{s}
\end{aligned}
$$

where $E_{s} I$ and $G_{n z} J$ are the bending and torsional rigidities of the reinforcement, respectively (see Fig. 3). To proceed and demonstrate the complete solution, consider a rectangular cross section for the reinforcement. Let $h$ denote the reinforcement thickness and $d$ the width. Then, the $I$ and $J$ values are found as

$$
\begin{aligned}
& I=\left(d h^{3 / 12}\right) \\
& J=\left[0.3 h^{3} d^{3} /\left(h^{2}+d^{2}\right)\right]
\end{aligned}
$$

Combining Eqs. (9-11), the following is derived:

$$
\begin{aligned}
G_{n z}\left(0.3 h^{3} d^{3} / h^{2} d^{2}\right) \bar{\kappa}_{s n} & =\psi \sin \alpha+\varphi \cos \alpha \\
E_{s}\left(d h^{3} / 12\right) \bar{\kappa}_{s} & =\varphi \sin \alpha-\psi \cos \alpha
\end{aligned}
$$

Eliminating $d$ from Eq. (12) leads to the condition for the shape of the neutral hole in terms of $\alpha(s)$ and $h(s)$ :

$\frac{0.3 G_{n z} 12^{3}(\varphi \sin \alpha-\psi \sin \alpha)^{3}}{E_{s}^{2} h^{8} \bar{\kappa}_{s}^{2}+12^{2}(\varphi \sin \alpha-\psi \cos \alpha)^{2}}=E_{s}(\psi \sin \alpha+\varphi \cos \alpha) \frac{\bar{\kappa}_{s}}{\bar{\kappa}_{s n}}$

The solution to Eq. (13), i.e., obtaining $\alpha(s)$ for a chosen $h$ (choosing the reinforcement thickness to be uniform and a certain multiple of the laminate thickness) will give the exact neutral hole shape. Finally, the reinforcement width distribution is determined from Eq. (12) as

$$
d(s)=\left[12(\varphi \sin \alpha-\psi \cos \alpha) / E_{s} h^{3} \bar{\kappa}_{s}\right]
$$

\section{Examples}

\section{Plate Under Bending Moments}

In this case, let the plate be under pure bending moments $M_{1}$ and $M_{2}$ applied at the edges of the plate whose normals are $x$ and $y$ axes, respectively. The well-known plate equilibrium equations give $M_{x}=M_{1}, M_{y}=M_{2}$, and $M_{x y}=0$. Then, from Eqs. (4-7) the functions $\varphi$ and $\psi$ are determined:

$$
\begin{aligned}
& \varphi=M_{2} x+c_{1} \\
& \psi=M_{1} y+c_{2}
\end{aligned}
$$




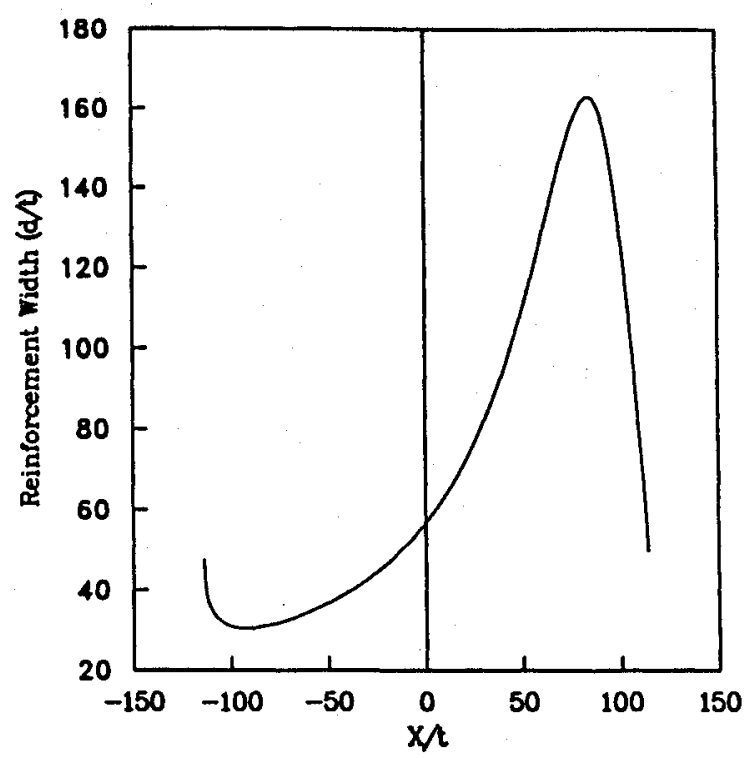

Fig. 4 Reinforcement distribution for moment loading.

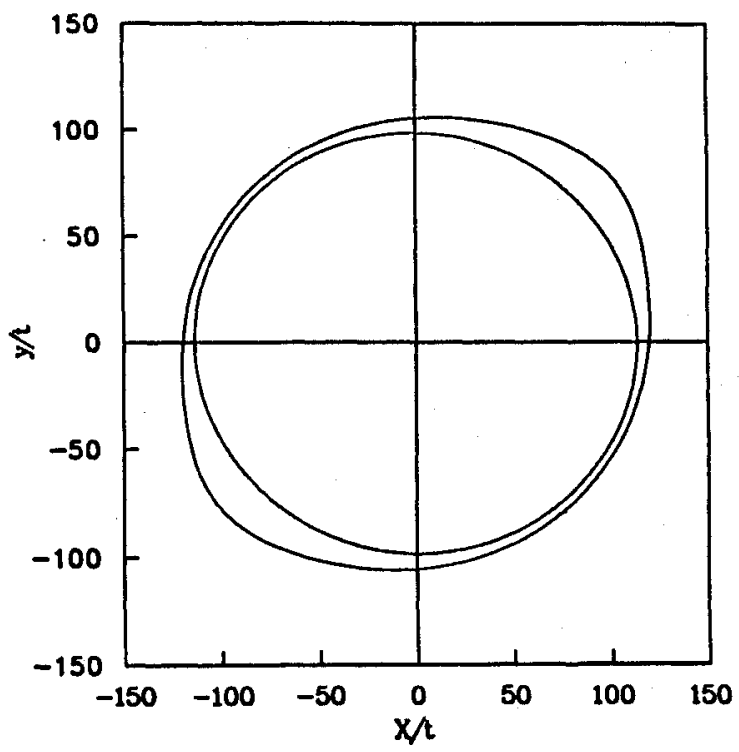

Fig. 5 Reinforcement distribution and hole shape.

where $c_{1}$ and $c_{2}$ are constants of integration. These can be chosen to be zero without loss of generality.

When Eq. (15) is substituted in Eq. (13) and after some algebraic manipulation, a seventh order polynomial in $\tan \alpha$ is obtained. The roots of this polynomial are obtained to describe the shape of the neutral hole. The procedure is as follows. A starting point in the plate is identified. Then the equations are cast into cylindrical polar coordinates where a radius vector $R(\theta)$ is chosen to describe the equation for the neutral hole as $R=R(\theta)$. At some initial point, $\boldsymbol{R}_{0}, \theta_{0}$ in the $\boldsymbol{R}-\theta$ plane the roots of Eq. (13) are used to obtain $d R / d \theta$. Then $\theta$ is incremented in a forward-difference scheme to obtain a new $R$. At this new $R, \theta$, the above step is repeated. Inspection of the terms in Eq. (13) case in the $R-\theta$ plane reveals that $R(\theta)=R(\theta+2 \pi)$, thus describing a closed hole. In fact, Eq. (13) can be used to determine the conditions under which a closed hole is possible. Once the neutral hole shape is determined, the reinforcement distribution is easily computed from Eq. (14). To demonstrate this procedure the following numerical example is presented.

Example: $[+40 /-40]_{2 s}$

Ply Properties: $E_{1}=133 \mathrm{GPa}, E_{2}=9 \mathrm{GPa}, G_{12}=3$ $\mathrm{GPa}, G_{23}=5 \mathrm{GPa}, \nu_{12}=0.26$.
Ply Thickness: $1 \mathrm{~mm}$.

Shape of Neutral Hole: The above-explained solution scheme is implemented to obtain $\alpha(s)$.

Reinforcement Distribution: From Eq. (14) with prescribed thickness (the thickness is chosen to be $16 \mathrm{~mm}$, which is twice the laminate thickness $t$ ).

Results: Presented in Figs. 4 and 5.

\section{Conclusions}

In this Note we have presented a method to design the shape of a cutout and the cutout edge reinforcement distribution for a symmetrically laminated plate, when it is remotely loaded by uniform bending moments. The case of planar loading for similar laminates was addressed elsewhere.$^{6.7}$

In the laminated plates we studied, the fibers are straight in each layer of the plate, and they are tangential to the edge of the cutout in the reinforcement. This is not a restriction, as the solution presented could very well accommodate other types of constitutive models for the reinforcement. Classical laminate theory to model the laminate and technical beam theory to model the reinforcement are used. In many practical constructions, it has been shown before that the bendingstretching coupling diminishes as the laminate gets thicker. We have developed the solutions for these unsymmetrical layups, ${ }^{7}$ and found that the material coupling has a small effect on the reinforcement distribution in many cases. Neglecting this material coupling and using the procedures developed in Ref. 6 leads to a solution for unsymmetrically laminated plates.

For the case of remotely applied moments, an exact solution within the context of classical laminate theory is presented. As mentioned in the introduction, all previous attempts to obtain a neutral hole have been done without accounting for flexural plate response, and assuming material isotropy. Here, both of these restrictions are relaxed. The solutions reported here are novel and their extension to the more complex case of laminated cylindrical panels and laminated shells is underway.

\section{Acknowledgments}

E. Senocak is grateful to the Turkish Government for the financial assistance through a Graduate Research Assistantship. The authors are appreciative of the support received from the Rackham Graduate School, University of Michigan, throughout this study.

\section{References}

${ }^{1}$ Lekhnitskii, S. G., "Anisotropic Plates," translated by S. W. Tsai, and T. Cheron, Gordon and Breach, New York, 1968.

${ }^{2}$ Lekhnitskii, S. G., "Theory of Elasticity of an Anisotropic Body," translated by P. Fern, and J. J. Brandstatter, Holden-Day, San Francisco, CA, 1963 .

${ }^{3}$ Savin, G. N., "Stress Distribution Around Holes," Nauka Dumka Press, NASA TT F-607, Kiev, Nov. 1968.

${ }^{4}$ Mansfield, E. H., "Neutral Holes in Plane Sheet-Reinforced Holes Which are Elastically Equivalent to the Uncut Sheet," Quarterly Journal of Mechanics and Applied Mathematics, Vol. VI, Pt. 3, London, 1953 , pp. 370-378.

${ }^{5}$ Richards, R., Jr., and Bjorkman, G. S., Jr., "Neutral Holes; Theory and Design," Journal of the Engineering Mechanics Division, ASCE, Vol. 108, Oct. 1982, pp. 945-960.

${ }^{6}$ Senocak, E., and Waas, A. M., "Design Considerations for Symmetrically Laminated Plates with Cutouts," AIAA 33rd SDM Conference Proceedings, TX, April 1992, pp. 3187-3197.

'Senocak, E., and Waas, A. M., "Neutral Holes in Laminated Plates," 6th U.S.-Japan Conference Proceedings, Orlando, FL, June 1992 (in print).

${ }^{8}$ Whitney, J. M., "Structural Analysis of Laminated Anisotropic Plates," Technomic Publishing, Westport, CT, 1987. 